\title{
Nathalie Luca (éd.), Quelles régulations pour les nouveaux mouvements religieux et les dérives sectaires dans l'Union européenne?
}

Aix-Marseille, Presses Universitaires d'Aix-Marseille, coll. « Droit et religions ", 2011, $188 \mathrm{p}$.

Émir Mahieddin

\section{OpenEdition}

\section{Journals}

Édition électronique

URL : http://journals.openedition.org/assr/24034

DOI : $10.4000 /$ assr.24034

ISSN : $1777-5825$

Éditeur

Éditions de l'EHESS

Édition imprimée

Date de publication : 30 décembre 2012

Pagination : 229

ISSN : 0335-5985

\section{Référence électronique}

Émir Mahieddin, « Nathalie Luca (éd.), Quelles régulations pour les nouveaux mouvements religieux et les dérives sectaires dans l'Union européenne? », Archives de sciences sociales des religions [En ligne], 160 | octobre-décembre 2012, mis en ligne le 18 mars 2013, consulté le 21 septembre 2020. URL http://journals.openedition.org/assr/24034 ; DOI : https://doi.org/10.4000/assr.24034

Ce document a été généré automatiquement le 21 septembre 2020.

() Archives de sciences sociales des religions 
Nathalie Luca (éd.), Quelles régulations pour les nouveaux mouvements religieux et les dérives sectaires dans l'Union européenne?

Aix-Marseille, Presses Universitaires d'Aix-Marseille, coll. « Droit et religions ", 2011, $188 \mathrm{p}$.

Émir Mahieddin

\section{RÉFÉRENCE}

Nathalie Luca (éd.), Quelles régulations pour les nouveaux mouvements religieux et les dérives sectaires dans l'Union européenne ?, Aix-Marseille, Presses Universitaires d'Aix-Marseille, coll. « Droit et religions », 2011, 188 p. 
1 Il est possible de l'entrevoir dans son titre même, cet ouvrage collectif dirigé par Nathalie Luca, apparaît comme un exercice de réflexion à l'interstice entre sciences interprétatives et sciences normatives. Il s'agit en effet des actes d'un colloque de janvier 2010 qui réunissait des chercheurs en sciences sociales, des juristes et des praticiens administratifs ayant pour intérêt commun la question de la gestion publique des problèmes éventuels engendrés par la recomposition des pratiques du croire en Europe. Les sciences sociales ont souvent perçu cette redistribution comme une phase de " retour du religieux », notamment avec l'apparition de ce qui a été désigné par l'expression consacrée de «NMR» (Nouveaux mouvements religieux); des collectifs que l'on rencontre plus fréquemment dans les controverses médiatiques sous le terme, aujourd'hui connoté péjorativement, de «sectes». Jean Baubérot l'a écrit dans la conclusion générale à l'ouvrage, mais il convient de le réaffirmer, l'organisation d'un tel colloque ayant pour thème un objet polémique représentait «une entreprise risquée et courageuse » (Baubérot, p. 178), et ce, à plusieurs titres.

2 En effet, cette initiative, au-delà de sa dimension controversée du fait des suspicions de l'opinion publique à l'égard des mouvements concernés, présentait aussi quelques difficultés d'ordre scientifique qui n'ont pas manqué d'être soulevées dans l'ouvrage à plusieurs reprises. Le choix a d'ailleurs été fait de restituer les débats ayant eu cours durant le colloque, ce qui constitue non seulement une bonne manière de percevoir en partie les réflexions menées durant la journée dont ce livre constitue une trace écrite, mais aussi une solution élégante au souci de rendu dialogique, entravant ainsi le potentiel monopole d'un discours scientifique à prétention hégémonique. Les questions des adhérents des mouvements religieux - ou se réclamant du religieux -, des personnes se considérant comme «victimes" de ces mêmes mouvements, des journalistes, des avocats et des fonctionnaires - tous présents dans la salle - permettent en effet de saisir la complexité et la plurivocité en jeu quand est abordé un thème qui dépasse largement le champ des religions pour gagner les terrains du politique, du juridique et les dimensions identitaires et éthiques de toute tentative de régulation de ces nouveaux phénomènes sociaux.

3 L'Europe a pris connaissance des mouvements dits " sectaires » à partir de la fin des années 1970. Tout de suite, ces mouvements ont été associés à un certain nombre d'inquiétudes, relatives à des faits divers largement relayés et commentés par les médias de masse (suicides collectifs, attaques terroristes, allégations sur de supposées ramifications réticulaires au sein des instances de pouvoir, etc.). Cette inflation médiatique autour des « sectes » a fait naître un certain nombre d'inquiétudes au sein 
des populations, dont les médias sont probablement en partie à l'origine, mais dont ils se font, aussi, tout simplement l'écho. La réponse des pouvoirs publics a consisté en la création d'observatoires ou de dispositifs législatifs de régulation, qui sont passés dans les articles recueillis dans cet ouvrage, présentés par les acteurs qui y sont eux-mêmes investis. Sont ainsi évoqués différents modèles européens (français, suisse, belge, britannique, espagnol et italien) qui malgré leur diversité convergent sur certains points. Ainsi, dans sa contribution, Jean-Paul Willaime parle-t-il de la construction progressive dans la majeure partie des pays européens d'une "laïcité de reconnaissance » (p.23), à savoir une laïcité qui respecte ses principes fondamentaux sans s'interdire de prendre en compte de manière officielle le rôle des institutions et/ ou organisations religieuses dans la vie publique. C'est de ce souci de gestion et de pluralisme qu'émerge une des lignes de tension structurantes des travaux menés par les instances de régulation élaborées dans la contrainte de trouver la juste voie entre une gestion assurant la protection des individus (notamment dans leur dignité physique, psychologique et matérielle) sans empiéter sur la liberté du culte. Cette double injonction mène les acteurs concernés à mettre en avant un ensemble de principes éthiques (on parle durant le colloque de neutralité, d'impartialité, de recherche d'objectivité) dans la conduction de leurs missions d'information, de sensibilisation, ou de lutte contre les "dérives sectaires». Des postures adoptées découlent les méthodes d'observation et d'action à mener dans l'accomplissement du travail de régulation. Celles-là mêmes résultent bien souvent de facteurs qui varient en fonction des instances concernées, des traditions juridiques, politiques et religieuses, soit de manière générale, en articulation avec la profondeur historique de l'État-nation dans lequel elles s'inscrivent.

4 La multiplication des guillemets dans les lignes qui précèdent, de même que dans l'ouvrage, vient du souci partagé entre chercheurs et acteurs engagés de définir le cadre de leur activité et/ou de leur réflexion. Parmi les problèmes évoqués dans l'élaboration du discours sur les " dérives sectaires », le souci terminologique apparaît au premier plan. De quoi parle-t-on exactement ? Dès les premières lignes de l'ouvrage, Nathalie Luca évoque les difficultés posées par le langage, à commencer tout simplement par le choix du titre du colloque - et des actes dont il s'agit ici. Les notions de «nouveaux mouvements religieux » et de « dérive sectaire » font l'objet des débats tout au long du colloque et les discussions opèrent un véritable travail de cisèlement autour des notions-clés à mettre en avant. À partir de quel moment et selon quels critères légitimes peut-on parler de «dérive»? Les collectifs religieux, ou parareligieux, sont-ils plus susceptibles de "dérives " que d'autres? À quels types de mouvements doit-on s'arrêter? Les Églises historiques et les "grandes religions » (bouddhisme, islam, judaïsme, etc.) doivent-elles être observées et/ou surveillées au même titre que les organisations dites "sectaires »? Autant de questions dont les réponses ne sont pas si évidentes qu'elles pourraient le paraître, comme l'illustrent les discussions rapportées dans les débats qui jalonnent le recueil. Si la notion de " dérives " s'applique à toute « exigence du mouvement de traiter en interne ce qui est une atteinte aux lois et au droit » (p. 77), doit-on voir cela comme une particularité des mouvements de renouveau religieux, ou même au champ religieux ? Est-il légitime alors, de leur réserver des instances spécifiques? Si par ailleurs, la notion de lutte contre l'« emprise mentale " ressort comme un dénominateur commun des actions entreprises par les organismes de régulation, la question reste la même : parlons-nous toujours de dérives spécifiquement "sectaires"? Certains se refusent à différencier 
« religion » et « secte " par souci de neutralité, comme dans le cas suisse, d'autres vont jusqu'à parler dans la dénomination même de l'organisme d'observation de "sectes nuisibles », comme dans le cas belge. Le débat terminologique pourrait paraitre dénué d'importance à première vue, mais les termes ont un poids et des conséquences juridiques pratiques qui dépassent le cadre de pure élaboration théorique. Comme le rappelle F.Bellanger (p.119), l'enjeu du débat de définition est le suivant: si le mouvement considéré est une religion, alors aucune action juridique ne serait possible sans violer la liberté religieuse ; et ce n'est là qu'un exemple.

5 Au souci d'objectivité s'ajoute la volonté de ne pas blesser les différentes subjectivités par le discours, du fait, comme dit, de la nature polémique de l'objet dont traitent les discussions. Cet aspect émotionnel qui ressort dans les débats, avec retenue toutefois, notamment quand il s'agit de discuter des méthodes d'intervention jugées parfois trop coercitives ou des observations biaisées de la part des acteurs de régulation/ information. D'un côté comme de l'autre, la recherche de spectacularisation propre à la logique médiatique, de laquelle peuvent résulter des distorsions et de fausses vérités, complexifie un rapport déjà difficile entre les collectivités et les organisations religieuses. Les médias apparaissent d'ailleurs tout au long de l'ouvrage comme un acteur problématique, notamment du fait d'un postulat répandu, celui selon lequel il est supposé que «la visibilité seule reflète la typicité » (Barker, p. 127). Jean Baubérot enjoint néanmoins, dans sa contribution, de ne pas fustiger la presse, qui peut être le lieu de débats intéressants (même si, dans les mémoires, le spectaculaire l'emporte souvent sur les questions de fond), et fait appel à la responsabilité citoyenne de chacun.

6 Les médias se font porteurs, par la mise en avant de faits divers, de représentations collectives qui relèvent à la fois du souci sécuritaire, déjà évoqué, et d'une problématique identitaire dans le cas européen. Ils sont en cela, comme c'est remarqué dans la contribution de F. Bellanger de B. Knobel, un baromètre intéressant de l'opinion publique. Sur un continent dont l'histoire politique et les frontières étatiques ont été marquées, voire façonnées, par le principe cujus regio, ejus religio, il était probable que la prolifération de pratiques religieuses indépendantes des Églises institutionnelles suscite des réactions négatives, relatives à la préservation de l'unité nationale, souvent coextensive d'une religion. Malgré la sécularisation de la société, les pratiques légitimes ont, semble-t-il, marqué les esprits, renvoyant les nouveaux mouvements à de « fausses » religions et engendrant la méfiance vis-à-vis de ces derniers. Il arrive même que ces mouvements apparaissent comme des importations de l'étranger malgré une inscription locale parfois séculaire.

7 Quelles régulations pour les nouveaux mouvements religieux et les dérives sectaires dans l'Union européenne ne constitue qu'une étape de réflexion dans la continuité des travaux déjà menés par Nathalie Luca sur les problématiques sectaires, et annonce d'autres rencontres autour de cette thématique, souhaitées par les organisateurs eux-mêmes. Il offre une bonne vue d'ensemble des politiques européennes en matière de religiosités contemporaines. La perspective comparatiste permet d'aborder plus que la question de la régulation des « sectes » elle-même, elle autorise une compréhension du rapport du politique européen aux phénomènes religieux dans sa diversité tout comme dans son unité culturelle, même si tous les pays de l'Union n'ont pas fait l'objet d'une communication. Le cas de la Suède est évoqué par Nathalie Luca dans sa communication, et Jean Baubérot regrette dans sa conclusion qu'il n'ait pas pu être plus développé par les intervenants du colloque. Je me permettrais d'en dire quelques 
mots pour apporter des éléments de réflexion supplémentaires à l'ouvrage, de manière évidemment non exhaustive.

8 La Suède représente en effet un modèle original en Europe. Loin de discriminer négativement les nouvelles Églises et autres mouvements religieux minoritaires, le gouvernement suédois s'est récemment défait de son Église luthérienne d'État pour octroyer un statut légal à toutes les associations cultuelles. Ces dernières se voient même directement subventionnées par l'État suédois à hauteur du nombre de leurs adhérents. Si le modèle est intéressant de par son originalité, les polémiques médiatiques que connaît la Suède avec ses nouveaux mouvements religieux sont analogues à celles que l'on observe chez ses voisins européens (on pense notamment à l'affaire du double meurtre dans la congrégation pentecôtiste de Knutby, un fait divers qui a fait feuilleton à la télévision suédoise en 2004 et dont les rebondissements ont fait l'objet d'une pièce de théâtre à succès et d'un film au cinéma en 2009), auxquels s'ajoute depuis peu un débat quant à la légitimité de subventions publiques octroyées à des organisations qui véhiculent parfois des valeurs qui vont à l'encontre de celles promues par la démocratie nordique. Pour exemple, une émission télévisée a, en 2011, soulevé une polémique sur les positions homophobes d'un certain nombre de groupes chrétiens évangéliques (il s'agissait notamment de l'Armée du Salut). L'État peut-il se permettre de financer des acteurs dont les postures discriminent négativement des parties de la population alors que les politiques publiques prônent la tolérance envers ces mêmes minorités ? Loin de mettre fin à la problématique sectaire, les tentatives de régulation suédoises n'ont fait que déplacer les enjeux du débat. Reste à voir quels effets ce modèle récent donnera à long terme. Il conviendra, en tout cas, de continuer à observer ce modèle dans la quête d'un éventuel modèle européen unifié, et de l'intégrer dans les réflexions futures sur la problématique. 